\section{Adsorption erythrozytärer Antikörper}

K. Kleesiek ${ }^{1}$, C. Götting ${ }^{2}$, J. Diekmann ${ }^{3}$, J. Dreier ${ }^{4}$ und M. Schmidt $^{5}$

${ }^{1}$ Ehemaliger Direktor des Instituts für Laboratoriums- und Transfusionsmedizin, Herz- und Diabeteszentrum NordrheinWestfalen Ruhr-Universität Bochum, Bad Oeynhausen,

Deutschland

${ }^{2}$ MVZ Labor Limbach Nürnberg GmbH, Nürnberg, Deutschland

${ }^{3}$ Institut für Laboratoriums- und Transfusionsmedizin, Herzund Diabeteszentrum NRW, Bad Oeynhausen, Deutschland

${ }^{4}$ Herz- und Diabeteszentrum Nordrhein-Westfalen; Institut

für Laboratoriums- und Transfusionsmedizin, Universitätsklinik der Ruhr-Universität Bochum, Bad Oeynhausen, Deutschland

${ }^{5}$ Institut für Laboratoriums- und Transfusionsmedizin, Universitätsklinik der Ruhr-Universität Bochum, Bad Oeynhausen, Deutschland

Synonym(e) Differenzialadsorption; allogene Adsorption

Englischer Begriff allogeneic adsorption

Definition Die Adsorption erythrozytärer Antikörper ist die gezielte Elimination eines Antikörpers an seinem korrespondierenden Antigen.

Beschreibung Die allogene Adsorption erythrozytärer Antikörper ist eine Technik in der immunhämatologischen Diagnostik, die bei dem Vorliegen von freien Autoantikörpern (s. > Autoantikörper) Verwendung findet, wenn die Technik der ersten Wahl, die $>$ Autoadsorption, nicht durchgeführt werden kann. Darüber hinaus besteht die Möglichkeit, ein komplexes Gemisch verschiedener Alloantikörper durch selektive Elimination einzelner Spezifitäten zu entschlüsseln. Im blutgruppenserologischen Alltag kann die Kombination verschiedener $>$ Alloantikörper eine Antikörperdifferenzierung erheblich erschweren, wenn die meisten der eingesetzten Testerythrozyten aufgrund der Vielzahl verschiedener Alloantikörper positiv reagieren. Die Differenzialadsorption kann zur gezielten Entfernung einzelner Alloantikörper aus einem Alloantikörpergemisch eingesetzt werden. Die nach der Differenzialadsorption verbliebenen Alloantikörper können in der anschließenden Antikörperdifferenzierung eindeutig spezifiziert werden.

Bei dem Vorliegen einer Kombination von freien Autoantikörpern und Alloantikörpern besteht das Ziel der allogenen Adsorption darin, den Autoantikörper vollständig zu entfernen und den unbekannten Alloantikörper im Serum/Plasma zu belassen. Die zur Adsorption eingesetzten Erythrozyten müssen für das Antigen negativ sein, gegen das der Alloantikörper reagiert. Da der Alloantikörper unbekannt ist, setzt diese Technik voraus, dass verschiedene Erythrozyten mit bekanntem Antigenmuster parallel zur allogenen Adsorption eingesetzt werden. Alle Testerythrozyten eliminieren mit einem Aliquot der Serum-/Plasmaproben den Autoantikörper. Die Testzellen, die zur allogenen Adsorption eingesetzt werden, sollen in der Antigenverteilung so gewählt werden, dass die häufigsten transfusionsmedizinisch relevanten Antikörper erkannt werden. Folgende Antigene sollten bei der Auswahl der zur allogenen Adsorption eingesetzten Erythrozyten sowohl negativ als auch positiv ausgewählt werden: C-, Cw-, c-, D-, E-, e-, K-, Fy(a)-, Fy(b)-, JK(a)-, Jk(b)-, S-, s-, M-, N-, P1-, Le(a)-, Le(b)-Antigen.

Antikörper gegen hochfrequente Antigene werden mit der Methode „Adsorption erythrozytärer Antikörper" nicht erkannt, wenn alle eingesetzten Erythrozyten dieses Antigen tragen (antigenpositiv sind).

\section{Literatur}

American Association of Blood Banks (1999) Technical manual, 13. Aufl. S. Karger, Basel 